\title{
Light Fidelity: The Future of Data Communication
}

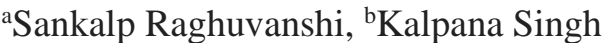 \\ aAmity School of Engineering and Technology, Amity University Noida \\ ${ }^{\mathrm{b}}$ Greater Noida Institute of Technology(affiliated to Guru Gobind Singh Indraprastha University ,Delhi)
}

\begin{abstract}
Light Fidelity (Lifi) is a mode of data transmission which is used to transfer data at a speed of more than 100 GB per sec. German Scientist Harald Hass coined the term light fidelity in the year 2011. currently optical fibers are used which can transfer data at speed of around $100 \mathrm{mbps}$ to $200 \mathrm{mbps}$, which is quite less than the light fidelity. Lifi makes use of light emitting diodes (LED) to transfer data at a lightning fast speed. with the help of Lifi, large number of files can be transferred parallel at a much higher transfer speed. This simultaneous transfer of data is made possible by electro-magnetic spectrum. In place of using the traditional radio frequency it makes use of much advance form ie. light to transfer data successfully. Advancement in light fidelity was made possible by development in the field of wireless communication. With the limitation of transfer speed and number of users increasing, radio waves are not able to fulfill the increasing demands

With the development of light fidelity scientist aims to remove most of the flaws while transferring data via radio waves. this new technology promises to transfer data at a lightning fast speed by changing the intensity of light waves. Lifi is the future of data transfer. The main objective of this paper is to give a brief idea about the Lifi and what all can be achieved with this futuristic technology.
\end{abstract}

Keywords- Digital communication, network traffic, wireless network, light fidelity, VLC, bandwidth, LED

\section{INTRODUCTION}

Lifi also known as light fidelity was invented by Harold Hass in year 2011. Lifi makes use of LEDs to transfer data form one device to another device without any interruption. with the help of LED, the data can be transferred at speed of more the $100 \mathrm{~GB}$ per second. In lifi the variation of light waves is even

faster than the flicker of an eye. Earlier wireless communication was done with the help radio waves. but the main demerit of using radio waves was that the speed of transfer was limited to $100-500$ mbps also if multiple files are being sent parallelly then the speed got reduced. hence with increase in the user relying on wireless transfer. It has become important to remove such flaws related to radio flaws. Hence light fidelity promises to overcome all problems related to security, accessibility, transfer speed etc.[4] Light fidelity can provide a much higher network speed as compared to wifi . since lifi make use of LEDS for data transfer as compared to wifi which make use of radio waves for data transfer . hence lifi can be able to achieve such a high speed while wifi cant.
For eg imagine downloading a complete HD movie in 30 sec or imagine uploading a large file on internet or youtube in fraction of seconds. such task can easily be achieved by lifi. Whereas if such tasks were given to wifi then it would take more then 2hours for completing the task . In today's world where millions of people are dependent in internet for there important works.light fidelity provide a efficient and easier way of communicating wirelessly. If we make use of radio waves then the speed of data transfer slows down if multiple devices are connected to a single network at once. Radio waves are smaller portion of electromagnetic spectrum which are used for data transfer using. Light fidelity provides a larger spectrum for transmitting the data as compared to traditions methodologies which uses radio waves for wireless communication.

The concept of light fidelity was first used by Herold Hass, a German physicist in his TED talks on the topic visible light.in the year 2011. He made use of a lamp which contain led bulb for transferring a short clip of a flower it was then projected on the screen. We also understand lifi in simpler language as a wireless network which uses light in place of radio waves for transferring data at a much higher speed. Lifi can solve the problem of transfer speed by increasing the overall bandwidth. It also delivers a extra band of frequency of order $400 \mathrm{THZ}$ while in case of radio waves it uses bandwidth of $300 \mathrm{THZ}$ which is quite less if compared to light fidelity.[6]

As we all know that radio waves are very dangerous to the health of a individuals. direct exposure to radio waves for a longer period of time can cause serious damage to various internal organs of the body. Light fidelity on the other hand is good for the body in the sense that it does not harm the internal organs of body. light fidelity has the potential of transforming the way we access the internet, downloading heavy files are just a click away, sending and receiving the mails are also made easy with the use of light fidelity.[1]

\section{ARCHITECTURE OF LIGHT FIDELITY}

The future of data transfer i.e. light fidelity is a great alternative to radio waves. it is much more secure. easy to use. Cheaper to handle etc. since it is a VLC (visible light communication) it makes use of spectrum of electromagnetic waves in the range of $400 \mathrm{THZ}$ to $800 \mathrm{THZ}$ for transmitting data and illumination. 
Light fidelity contains the following ----

1) LED light or bulb having high brightness which is used for transmitting data at higher speed.

2) As a receiving element, silicon photodiode can be used which has a good response to visible light source.

By switching the LEDS on and off rapidly can help to make combination of 1 's and $0 \mathrm{~s}$. by changing the rate of LEDs data can be encoded in light source and thereby new stream of data can be generated.

The major components of LIFI are as followed -----

1) light bulb

2) radio frequency power amplifier circuit

3) printed circuit board

4) enclosures

The PCB (printed circuit board) handles the electrical input and output. Of the light sources. Ii contain the microcontroller which is control various function of the lamp Fig (1). The radio frequency is created with the use of power amplifier and is sent to the electric field of bulb. [16].

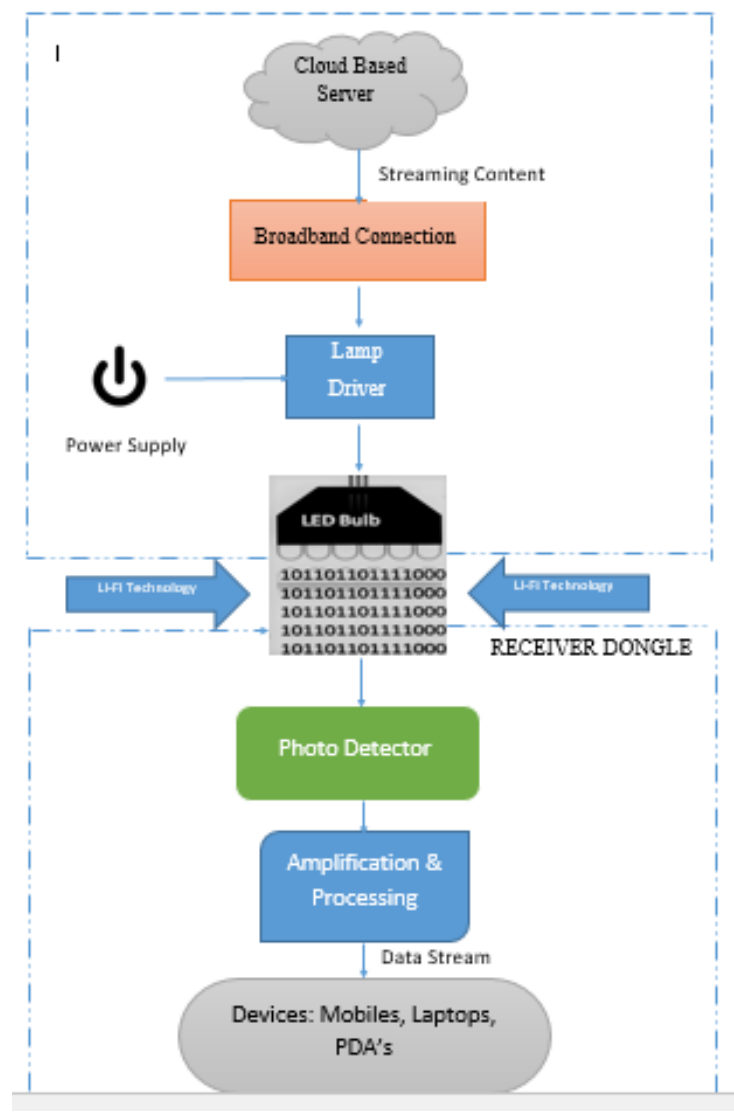

Fig.1. Mechanism of Li-Fi

\section{THE DIFFERENCE BETWEEN LIFI AND WIFI-}

3.1) The full form of LIFI is light fidelity whereas full form of WIFI is wireless technology.
3.2) lifi was discovered by the scientist Harald hass in the year 2011. Whereas WIFI was discovered by the NCR corporation in the year 1991 .

3.3) lifi is much more secure then the WIFI since the wall usually block the light sources hence it provides much more security of data.

3.4) distance of coverage is limited to 10 meters while distance of coverage is up to 32 meter in case of WIFI.

3.5) light fidelity is able to transfer data at a speed of $100 \mathrm{~GB} / \mathrm{s}$. whereas WIFI is capable of sending the data at a speed of $100-500 \mathrm{MB} / \mathrm{s}$ which is relatively very less then light fidelity.

3.6) light fidelity uses electromagnetic spectrum to transfer data from one device to another. Whereas WIFI make use of radio waves to transfer data from device to another.

3.7) the range of transfer of data from device to another is less as compared to WIFI in which range of transfer of data is much larger.

3.8) the overall cost of light fidelity is quite less if we compare it with the WIFI.[13]

3.9) lifi has a higher power availability as compared to WIFI.

3.10) lifi is used in airlines, exploration, underwater etc. whereas WIFI is used in browsing internet with hotspot etc.

\begin{tabular}{|c|c|c|c|}
\hline S.No. & Parameter & Li-fi & Wi-fi \\
\hline 1. & Speed & $>1 \mathrm{~GB} / \mathrm{s}$ & Around $150 \mathrm{mb} / \mathrm{s}$ \\
\hline 2. & Medium of data transfer & Use light as carrier & $\begin{array}{c}\text { Use radio } \\
\text { spectrum }\end{array}$ \\
\hline 3. & Spectrum range & $\begin{array}{c}\text { Visible light has } \\
10000 \text { times more }\end{array}$ & $\begin{array}{c}\text { Having less } \\
\text { spectrum range } \\
\text { than VLC }\end{array}$ \\
\hline 4. & Cost & Cheaper & Expensive \\
\hline 5. & Network topology & Point-to-Point & Point-to-Point \\
\hline 6. & Operating Frequency & $\begin{array}{c}\text { Hundreds of Tera } \\
\mathrm{Hz}\end{array}$ & $2.4 \mathrm{GHz}$ \\
\hline
\end{tabular}

Table 1. LIFI vs WIFI

\section{APPLICATION ON LIGHT FIDELITY}

4.1) Hospital - Since LIFI does not intervene with the devices using radio frequency it can be used in hospital for various applications. Li-Fi can be used in patient room, waiting area, operation room etc. doctors can also use LIFI to monitor and check the report of progress of patients easily.

Apart from hospitals it can also be used by pharmacists to receive the prescriptions directly. Lifi can also be used to track the prescribed medicines from the manufactures. The employees of hospital like nurse can directly check the status without going to the ascetic unit. Hence $\mathrm{Li}$-Fi can ease the work of both pharmsist and management of hospital. [3]

4.2) Retail outlets/store - light fidelity can help the customers to get information about various products 
available in the store. it can also help the customer to find their way in the store.

4.3) Traffic lights - light fidelity can be helpful in monitoring the real time traffic. this will help to solve the problem of frequent traffic jams and reduce the amount traffic on roads. Fig.(2)

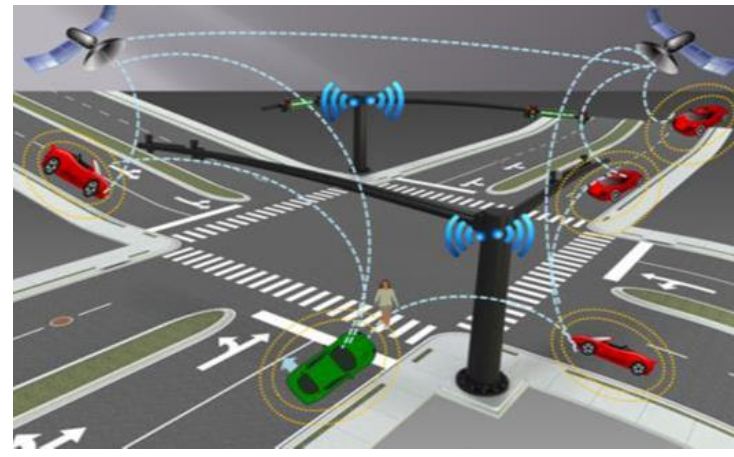

Fig 2: Traffic signals being controlled by LIFI technology

4.4) underwater --- light fidelity can be also be used under water. unlike WIFI which cannot be used underwater lifi can be efficiently used under water.[10] Fig. (3)

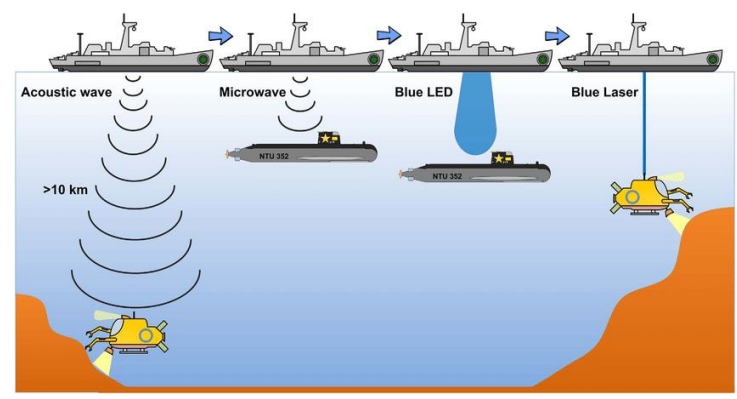

Fig 3: Underwater communication using LIFI technology

4.5) Aeroplanes - light fidelity can enable the passengers of airline to operate internet on the go. currently the per mb cost of using internet on the go is very costly. light fidelity promises to reduce the cost of operating the internet by a significant margin.

4.6) petrochemical carrier - cellular signals can cause spark or short circuits if used near to petrochemicals. But Li-Fi does not cause any harm and can be safely used without any worry of short circuit or explosion.

4.7) Wearables- Li-Fi can also be used in connecting smart clothes with LED lights.

4.8) Science exhibition - we can use light fidelity in science exhibition to find information about various working/nonworking science models.[2]

\section{CHALLENGES AND LIMITATIONS OF LIGHT FIDELITY}

5.1) The main disadvantage of using light fidelity is that it can only be used in presence of direct light

5.2) For a successful transfer of data from sender to receiver, there should be no obstruction between them.
5.3) Light fidelity cannot be used for long distance coverage. It can be only used for short distance coverage.

5.4) The mode of communication used in light fidelity is point to point communication because it makes use of high frequency which helps in short distance communication.[9]

5.5) Implementation of light fidelity requires totally different infrastructure as compared to other modes of communications.

5.6) $24 \times 7$ the light needs to be turned on if internet has to be used this will waste lot of electricity.[5]

5.7) Installation of light fidelity can only be done in limited areas since constant light is to be present between sender and receiver.

\section{6)METHODOLOGY}

There are of two parts of Li-Fi transmitter and receiver. We make use of led lights as a signal source between the two end points. modulated lights usually carry data from the LEDs. The data is modulated and demodulated between the transmitter and receivers by the microprocessor unit (MPU). The input signals are modulated by the transmitter at required time period. The data is then transmitted in form of 1 's and 0 's by the use of LEDs. The flashes are caught by the receiver with the help of photodiode. It then amplifies the signal to produce the required output. White LED light implements the light fidelity at the downlink transmitter. We can also use a sequence of LEDS for transferring data parallelly and LED of different color to change the frequency of the LED for data encryption of different frequency.[7]

\section{USING LIFI TECHNOLOGY FOR MONITORING COVID-19 PATIENTS}

Nav wireless Technology has adopted the technology of lifi to send the important data of patients like reading temperature, ventilators etc wirelessly by LED bulbs. This technology can be a major breakthrough for the doctors who got exposed while visiting the covid patients even after using the protective gears.

Since use of mobile phones and WIFI are restricted near the covid 19 patients, hence monitoring the patients remotely is also not possible.

Since there is no usage of radio signals or the electromagnetic signals in order to send data. This technology is completely safe for treating the covid 19 patients. The medical equipment sends the data and it is received by the light and the reverse of this mechanism is also possible.

One more benefit of using lifi technology during treatment of Covid 19 patents is that the data and report of infected patents are secured since no one will be able to access them unlike WIFI. 


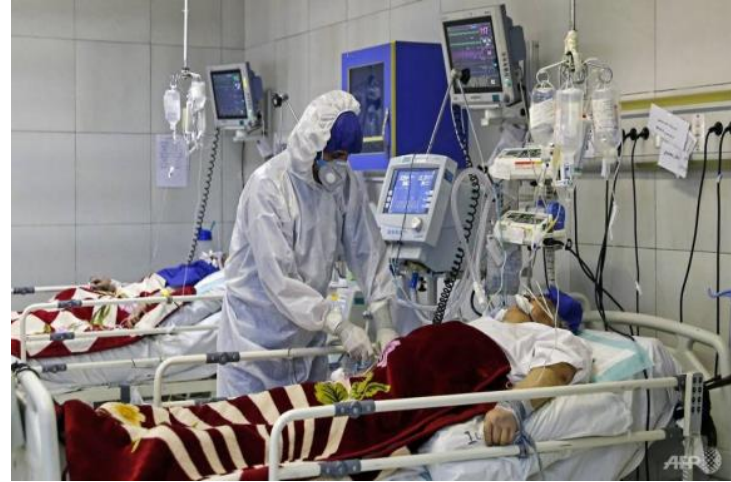

Fig 4:covid 19 patient being treated in a hospital

\section{WORKING PRINCIPLE OF LI-FI}

white led bulbs are used to implemented light fidelity with the help of downlink transmitter. If a constant current is applied then the devices can be used for illumination. But if current is altered at a fast speed or rate then variation of optical output can take place at a high speed. The overall operation is quite easy - transmission of digital 1 if LED is on but if led is switch off then you transmit digital 0 . transmission of data can take place by switching the LED on and off at a rapid speed. [8]

Several modification or improvement can be done by the use of sequence of of LEDs for transmitting the data parallelly or by the use of different colored LEDs like red, green, blue to change the frequency of the light. by doing such modification we can get a high speed of 20GBPS. Imagine downloading a full hd movie in under 40sec or even lesser. Such promising speed can be offered by the light fidelity.

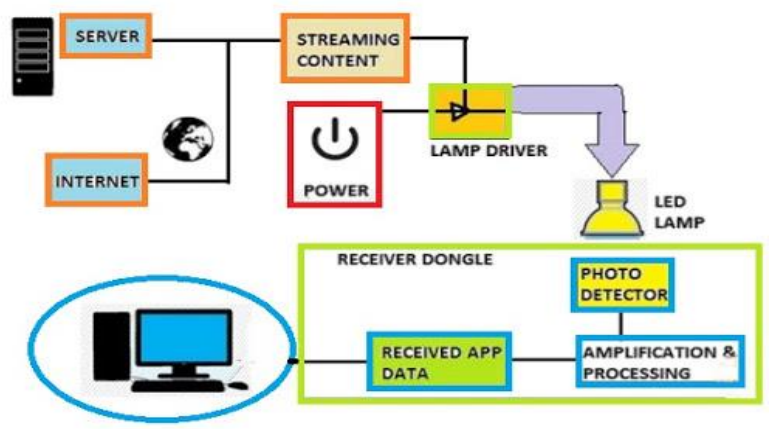

Fig.5 working involved in lifi technology

\section{RECENT ADVANCEMENT IN THE FIELD OF LIGHT FIDELITY}

\section{1) light fidelity for smart cities and town-}

Lifi can be used in town and cities to transmit data wirelessly with the help of LEDS bulbs and it can be used in street lights to make the town smart and efficient.

9.2) topology will be the most important thing in future-

Researchers from all over the world have predicted that in future the data transfer form one device to another will be of much higher speed as compared to today's network speed. But with this high speed the amount of complications will also increase and due which could obstruct the transfer speed. Hence light fidelity aims to remove all the complications without effecting the speed of data transfer. Hence the demand of such networks will also increase in densely populated areas .[12]

\section{3) Reliable communication and network improvement---}

Light fidelity aims to provide high speed data transfer between multiple devices inside a limited area of coverage. it is seen often in WIFI that if too many devices are connected to common wireless network then the speed is distributed among various devices due to which the speed offered to individual devices became very less. For example if the network is capable of providing a speed of $100 \mathrm{MB} / \mathrm{s}$ and there are 10 devices connected to the network then the speed gets distributed within 10 devices and each of the device will get a speed of only $10 \mathrm{MB} / \mathrm{S}$ which is very less if we compare it to $100 \mathrm{MB} / \mathrm{S}$ which is provided by the network.

While in case of light fidelity if the speed of $100 \mathrm{~GB} / \mathrm{S}$ is being provided and 10 devices are connected to the network then each of device will get a speed of 100GB. rather 10GB/s which would be provided in case of WFI.

\section{4) high speed data transfer}

Light fidelity can provide a much higher network speed as compared to WIFI. since lifi make use of LEDS for data transfer as compared to WIFI which make use of radio waves for data transfer. hence lifi can be able to achieve such a high speed while WIFI can't.

For e.g. imagine downloading a complete HD movie in 30 sec or imagine uploading a large file on internet or YouTube in fraction of seconds. such task can easily be achieved by lifi. Whereas if such tasks were given to WIFI then it would take more then 2 hours for completing the task. [15]

\section{DESIGN OF LIGHT FIDELITY}

Light fidelity contains a number of LEDs and lamp. There are many wireless devices like laptops, smartphone, PDA

There are multiple factors that must be taken into account like----

1) light should be present

2) light of sight (LOS)

3) for improving the overall performance, we can use fluorescent light and bright LEDs light

4) data received by the photo detector.

\section{VISIBLE LIGHT COMMUNICATION}

Radio waves were earlier used for transferring data from one device to another. but transfer speed was very limited it also lacked security reliability and was much expensive to use. infrared on the other hand can damage the eyes if not used at a low power. whereas ultraviolet rays can only be used in the places free from human since it could prove to be really 
harmful for humans. Hence visible light can be used for data communication since there is no harmful effect involved in visible light.

It provides much larger bandwidth and is even safer to use as compared to radio waves. VLC is a type of medium used for data communication, which make use of visible light which ranges from 400THZ to $800 \mathrm{THZ}$. [11]

\section{FEATURES OF LIGHT FIDELITY}

10.1) Bandwidth:

The bandwidth of light fidelity is much more then radio waves. This makes it much more popular to use as compared to WIFI.

\section{2) Data Density}

Data density of lifi is much more then radio waves. It is seen that data density of lifi is 1000 times more than the WIFI. It is seen that radio waves usually suffers from the problem of interference whereas visible light can be stored in light illumination

\section{3) Hight speed}

Lifi can be used to achieved a high-speed data transfer. this transfer of data is usually free from interreference. it also has a large bandwidth as compared to WIFI which has a comparatively smaller bandwidth.

\section{CONCLUSION-}

A thorough market research suggest that light fidelity will hit the market value of 900 million USD in 2020.

Light fidelity is a great technology which has the power or ability to transform the whole wireless technology. the features which are offered by light fidelity are totally new and advance. although there are very few items of Li-Fi currently in the market, but because of its merits it will deferentially be an instant success in the market.

There are various industries and organizations which are currently testing the infrastructure involved in light fidelity. many organizations like google, Facebook and apple have started testing the light fidelity for its implementation in their future devices.

Although there are various features related to light fidelity which makes it completely unique but there are several limitations involved which must be solved in order to get full utilization of this technology. [14]

\section{FUTURE SCOPE}

Even in today's time most of the people have very less knowledge and even some of them have never heard about light fidelity. even now this technology has not been implemented fully. But once it is ready to be used properly it would be able to solve most of the network related queries/problems and also resolve most of the conflicts present in today's wireless networks.
Recent study has proved that light fidelity technology can be used for covid patients. since the lifi make use of led bulbs for communicating effectively it can prove to be successful in protecting the data and reports of patients unlike WIFI.

Radio frequency cannot be used in multiple field and area. This is one of the major drawbacks of radio frequency. for example, radio frequency can be dangerous in hospital. RF can be also disturb calibration and reading of the devices. Like MRI machine etc. Since LIfi does not use radio frequency for transmitting data from one device to other. Hence LiFi can be alternative to RF and it can also be used in hospital.

LiFi can also be used by scientist for preforming various scientific studies and experiments. it is often seen that many times scientist require a much secure reliable and faster network for there experiments and indentations. But traditional radio waves are not at all secure to be used. they can easily become the target of hackers. since the range of signals is much wider hence hackers can easily hack into the delicate information on users. Whereas the much more advance form of network i.e. light fidelity is much more secure to be used. since the range of network coverage is limited the hackers have to sit really close to the network which is not possible. hence shorter coverage area can be used as an advantage for the light fidelity.

Apart from this light fidelity provide much more advance features which can be used in the advancement of technology and science. All these features make the lifi unique and somewhat different from the traditional radio waves. in today's time people are mostly annoyed by the speed of network, steep prices, limited availability of data. Light fidelity promised to remove all such problems which radio waves fails to do.

1)Light fidelity is much cheaper to use as compared to traditional radio wave.

2) light fidelity provides data transfer speed of $100 \mathrm{~GB} / \mathrm{S}$.

3) Light fidelity is much more secure to use as compared to traditional radio waves.

All these problems which were faced by the common people are easily solved by light fidelity.

$\mathrm{RF}$ are not used in military operations but LiFi can became a alternative to RF even in military operations. One of the threats to wireless communication is lack of proper security and protection of your data. It is the most crucial problem which is not addressed in WIFI. Lifi has the ability of providing complete data protection as well as privacy to the user data. The implementation of lifi can also be done by US navy in their submarines to enhance and support the submarine communication system. Radio waves can also not be used for data transmission underwater. The radio waves can also harm the waves. Hence it is not advisable to use radio waves underwater. $\mathrm{Li}-\mathrm{Fi}$ does not harm the sea waves and also provide successful data transmission between devices. Hence $\mathrm{Li}-\mathrm{Fi}$ is the best alternative to radio frequency and will prove to be a great invention in the field 
of science. Light fidelity has the potential of becoming the major game changer in the field of data communication. Continuous research is being done even now to rectify and sort of problem or error in this new and advance form of technology and also to remove or fix the limitations of light fidelity.

\section{REFERENCES}

[1] Haas, Herald (July 2011).”Wireless data from every light bulb". TED Global. Edinburgh .Scotland

[2] Soltani, MD, Arfaoui, MA, Tavakkolnia, I. et al. Bidirectional optical spatial modulation for mobile users: towards a practical design for lifi systems. arXiv preprint arXiv:1812.03109 (2018)

[3] Partha Pratim Shome, Kaushtubh Dutta, "Li-Fi: A Potential Game Changer in the History of Data Transfer", International Journal of Electrical, Electronics and Data Communication, Vol.4, issue: 12, Dec: 2016.

[4] S. Shao, A. Khreishah, and H. Elgala, "Pixelated VLCBackscattering for Self-Charging Indoor IoT Devices," IEEE Photonics Technology Lett., vol. 29, no. 2, 2017, pp. 177-80

[5] A. M. Sonnad, A. Gopan, Sailakshmi N R, Divya S, Ambika R, "Recent Advancements in LI-FI Technology", International Journal of Electrical, Electronics and Data Communication, vol. 1, issue. 10, pp. 61-63, Dec. 2013.

[6] Valiveti HB, Polipalli TR. Light fidelity handoff mechanism for content streaming in high speed rail networks. In: 2017 8th IEEE Annual Information Technology, Electronics and Mobile Communication Conference (IEMCON). IEEE; 2017:488-492.

[7] Alomari, SA: A novel adaptive caching mechanism for video on demand system over wireless mobile network. arXiv preprint arXiv:1812.08309 (2018)
[8] M.S. Islim and H. Harald, 'Modulation techniques for Li-Fi', in: ZTE Commun, Vol. 14, 2016, pp. 29-40. doi:10.3969

[9] A. Jovicic, J. Li and T. Richardson, Visible light communication: opportunities, challenges and the path to market, IEEE Communications Magazine 51(12) (2013), 26-32, ISSN 01636804. doi:10.1109/MCOM.2013.6685754.

[10] http://www.ijsrp.org/research-paper-0416/ijsrp-p5275.pdf

[11] D. Karunatilaka, F. Zafar, V. Kalavally, and R. Parthiban, "LED based indoor visible light communications: State of the art," IEEE Commun. Surveys Tuts., vol. 17, no. 3, pp. 1649-1678, 3rd Quart., 2015. doi: 10.1109/COMST.2015.2417576.

[12] Liju Sajan, Lince Mathew, Abraham Thomas, "Wireless data transfer using visible light communication." IJRET: International Journal of Research in Engineering and Technology eISSN: 23191163 | pISSN: 2321-7308

[13] Jitesh Nagdev, Dipesh Sher, Rohit Nathani, Gaurav Kalwani ,Wireless data transfer using light fidelity International Journal of Science and Research (IJSR), India Online ISSN: 2319-706.

[14] Sharma, R.R., Raunak, Sanganal, A. 'Li-Fi Technology: Transmission of Data Through light" International Journal of Computer Technology \& Applications, Vol. 5 (1).

[15] Y. Khare, V. P. Tiwari, A.B. Patil, K. Bala, "Li-Fi Technology, Implementations and Applications", International Research Journal of Engineering and Technology, Vol 3, Issue 4, pp. 1391 1394, April 2016

[16] Varun Shah, Sumit Chaudhary, Bhavesh Jain "Architecture based on $\mathrm{Li}-\mathrm{Fi}$ to enhance the working environment", International Journal of Advanced Research in Computer and Communication Engineering, Vol. 6, Issue 2, February 2017 